\title{
Dividing attention between color and shape: Evidence of coactivation
}

\author{
J. TOBY MORDKOFF \\ Center for Human Information Processing, University of California, San Diego, La Jolla, California \\ and \\ STEVEN YANTIS \\ Johns Hopkins University, Baltimore, Maryland
}

\begin{abstract}
When attention is divided between spatially distinct objects, the time to detect a target decreases when two or more targets are present. This redundancy gain can be accounted for by an interactive race model (Mordkoff \& Yantis, 1991) in which separate decisions are made about each object, but environmental contingencies among the objects can influence decision times. In the present study, we examined whether the model also accounts for performance when attention must be divided between stimulus attributes other than spatial location. Subjects made targetpresent responses when displays included a prespecified color, a prespecified letter, or both target features. The data violated the predictions of all separate-activations models, including the interactive race model. Two control experiments ruled out an alternative account based on task complexity. We conclude that coactivation occurs when target attributes from two separable dimensions are simultaneously present, but not when target attributes come from the same dimension. A modular hybrid of race and coactivation models is tentatively proposed.
\end{abstract}

When attention is divided between two visual objects, one may ask how the information accumulated from these multiple sources comes to produce a single response. This question can be directly addressed through the use of the redundant-target detection task (e.g., Miller, 1982; Mordkoff \& Yantis, 1991; Mordkoff, Yantis, \& Egeth, 1990; van der Heijden, La Heij, \& Boer, 1983). This task requires subjects to press a button (thereby making a go response) when they detect the presence of one or more targets and to do nothing (no-go) when no targets are presented. The experiments are designed to test whether two targets may be identified at once. Response time (RT) is the dependent measure, and the crucial analysis concerns the difference in the time required to make a response when the display contains two targets, relative to when the display contains only one target. An RT advantage for redundant-target trials (i.e., trials with two targets)-which is known as a redundancy gain or the redundant-signals effect-is taken as evidence of parallel processing.

This research was supported by Grants R01-MH43924 and T32MH14268 from the National Institutes of Mental Health and Grant JRINCA2-413 from the National Aeronautics and Space Administration. It is based in part on a doctoral thesis submitted to the Johns Hopkins University by the first author. A preliminary report was made at the 32nd Annual Meeting of the Psychonomic Society, San Francisco, CA. We thank Bill Bacon, Howard Egeth, Charles Eriksen, Jeff Miller, Cathleen Moore, Tram Neill, and Jeremy Wolfe for their advice and comments. We also thank Dana Feinberg, Jackie Ferris, Rima Kliore, and Danielle Picher for their aid in conducting the experiments. Correspondence should be sent to J. T. Mordkoff at the Center for Human Information Processing, University of California, San Diego, La Jolla, CA 92093-0109 (e-mail: furball@ucsd.edu).
Previous investigations of divided attention using the redundant-target detection task have led to two classes of models that can account for the redundant-signals effect (e.g., Grice, Canham, \& Boroughs, 1984; Miller, 1982; Mordkoff \& Yantis, 1991; van der Heijden, Schreuder, Maris, \& Neerincx, 1984): separate-activation, or race, models (e.g., Raab, 1962) and coactivation models (e.g., Miller, 1982). Both classes of models incorporate parallel processing to the level of target identification; they differ in their assumptions about how many targets may participate in response activation simultaneously. According to race models, each of the targets on redundant-target trials provides an independent opportunity for a targetpresent response to be triggered. Within race models, information is never combined across perceptual channels and only one target on redundant-target trials is directly responsible for the observed response-hence the alternative label, separate activations. These models explain the redundant-signals effect in terms of probability summation or statistical facilitation: With two targets, there are two opportunities for a rapid target identification and, thus, a rapid response.

In contrast, according to coactivation models, information concerning any and all targets may simultaneously contribute activation toward a target-present response. Therefore, on redundant-target trials it is possible for both targets to be partially responsible for the observed response. This class of models explains redundancy gains in terms of energy summation: With two targets contributing activation toward the same threshold, the response is activated more rapidly than when there is only one target contributing activation. 
Both classes of models are consistent with the redundantsignals effect in mean RT. However, the two model classes can be discriminated by examining the entire distribution of RTs. In particular, Miller $(1978,1982)$ has shown that all race models must satisfy the following rule, known as the race-model inequality:

$P\left(\mathrm{RT}<t \mid \mathrm{T}^{1}\right.$ and $\left.\mathrm{T}^{2}\right) \leq P\left(\mathrm{RT}<t \mid \mathrm{T}^{1}\right)+P\left(\mathrm{RT}<t \mid \mathrm{T}^{2}\right)$,

where $t$ is time, and $\mathrm{T}^{1}$ and $\mathrm{T}^{2}$ refer to targets in locations 1 and 2 , respectively; thus, $P\left(\mathrm{RT}<t \mid \mathrm{T}^{1}\right.$ and $\left.\mathrm{T}^{2}\right)$, for example, is the probability that a response has been made by time $t$ given redundant targets. The results from early tests using this method (e.g., Grice et al., 1984; Miller, 1982; van der Heijden et al., 1984) provided ambiguous support for both model classes. Sometimes the race-model inequality was violated (e.g., Miller, 1982, Experiment 4)-a result that favors coactivation models-and sometimes it was not (e.g., van der Heijden et al., 1984).

\section{Interactive Race Model}

Recently, we proposed a new model that combines ideas from both independent race and coactivation models (Mordkoff \& Yantis, 1991). This interactive race model incorporates two basic assumptions: (1) each target in a display provides a separate opportunity for a target-present response to be activated (i.e., the interactive race model is in the class of separate-activations models), and (2) correlations or contingencies among stimuli or between nontarget stimuli and whether a response should be made may affect the speed of perception and decision, respectively (which, in turn, may affect RT). This second assumption states that information in the perceptual channels may interact, but only to the extent that an informative contingency exists among elements in the experimental design (cf. the concept of internal constraint discussed by Garner, 1962). In a series of experiments, we independently manipulated two types of contingency and found evidence to support the interactive race model. Analysis of previous experiments showed that the conflicting support for separate-activations and coactivation models could also be explained by reference to the contingency-sensitive mechanisms that the interactive race model includes; experiments that produced violations of the race-model inequality included contingencies within their designs that favored redundant-target trials (i.e., they contained $b i$ ased contingencies; see Mordkoff \& Yantis, 1991, for details).

An important aspect of the interactive race model is that it reduces to being a simple race model when no biased contingencies are included in the experimental design. The model may be thought of as an independent race model to which two sets of contingency-sensitive mechanisms have been added; when no contingencies are present in the experimental design, these mechanisms become irrelevant and the model is equivalent to an independent race model. Thus, under experimental designs with no biased contingencies, the model predicts that the race-model inequality will always be satisfied. This prediction has also been previously confirmed (Mordkoff \& Yantis, 1991, Experiments 1 and 4; van der Heijden et al., 1984).

\section{Dividing Attention Between \\ Separable Dimensions}

Most experiments using the redundant-target detection task have examined the effects of dividing attention between spatially distinct objects. In particular, subjects usually have been assigned exactly one target (typically an uppercase letter) and have been required to respond "target present" whenever this letter appears one or two times within a display containing one or two letters (e.g., Grice et al., 1984, Experiments 3-5; Miller, 1982, Experiments 4 and 5; Mordkoff \& Yantis, 1991; van der Heijden et al., 1984).

In contrast, we have found very few studies examining the redundant-signals effect when visual attention must be divided between stimulus attributes other than spatial location. ${ }^{1}$ In such a task, subjects would be assigned a target feature within each of two separable perceptual dimensions, such as color and shape. A go response would be correct if either or both target features were presented; no response would be correct if neither target was presented.

There is some reason to think that dividing attention between nonspatial stimulus features might involve mechanisms different from those involved in dividing attention between two spatial locations or spatially disparate objects. Duncan (1984) was among the first theorists to distinguish between two possible representational bases for visual selection. Space-based accounts of visual selection assume that attention is directed to a circumscribed spatial region, such that all objects or attributes within that region are attended and everything outside it is not. In contrast, object-based accounts of visual selection assume that a perceptual display is preattentively organized into perceptual objects, and that attention is then directed to these objects on the basis of their properties and the demands of the current visual task. There is a great deal of evidence that, under some circumstances, attention appears to be distributed within a space-based representation. It is equally clear, however, that object-based representations will play an important role in comprehensive theories of vision (for discussions, see Duncan, 1984; Kahneman, Treisman, \& Gibbs, 1992; Kanwisher \& Driver, 1992; Yantis, 1992).

To the extent that object-based theories of visual selection characterize the performance of redundant-target tasks, one might predict that when attention is divided between two different objects, perceptual information about each one will be kept separate, but when attention is divided between stimulus attributes that are not clearly parts of distinct objects, perceptual information might be combined in some way. This leads us to the possibility that the predictions of the interactive race model in particular, and separate-activations models in general, might be confirmed when attention is divided between two different objects (as in most previous studies of the redundancy gain) but disconfirmed when the signals to which 
attention is directed are not parts of spatially disparate objects. In particular, coactivation may occur when the two target features are parts of a single object. One purpose of the experiments reported in this article was to test this possibility. Alternative factors that might lead to coactivation will be discussed after the first experiment.

\section{EXPERIMENT 1}

In Experiment 1, subjects were required to divide their attention between the separable dimensions of color and shape, with all stimulus features being parts of a single object. The task was go/no-go target detection, requiring a buttonpress if the color green, the shape $\mathrm{X}$, or both target features were present, and no response if neither target was present. A single stimulus was presented on each trial: a colored letter at fixation. In this case, single-target displays included a purple $X$ or a green $O$, and redundanttarget displays always included a green $\mathrm{X}$. According to our conjecture that redundant target features from a single object may coactivate a response, we should here observe violations of the race-model inequality.

\section{Method}

Subjects. Twelve undergraduate students at The Johns Hopkins University and the University of California, San Diego, participated as part of an introductory course requirement. All subjects reported normal or corrected-to-normal acuity and no color impairment.

Apparatus and Stimuli. The stimuli were presented on Princeton Graphics SR-12 color monitors controlled by Sigma Design Color -400 graphics adapters or on NEC Multisync color monitors controlled by VGA graphics adapters. The subjects responded by pressing a button on a custom response box placed directly in front of the screen. Each subject used the dominant index finger to respond.

Each display included one colored letter presented against a black background. The letters (X, $\mathrm{O}$, and I) and colors (green, cyan, and purple; 4-bit palette codes 10,11 , and 13) were chosen for their low confusability, as well as the symmetricality of the letters and the similar brightness of the colors. From a viewing distance of $45 \mathrm{~cm}$, the letter subtended $1.40^{\circ} \times 0.89^{\circ}$ visual angle.

Design. All of the experiments reported in this paper, including Experiment 1, used a design without informative contingencies, equivalent to the design used in Experiment 1 of Mordkoff and Yantis (1991). Each block included approximately 50 trials: 42 test trials, with 5 randomly selected warm-up trials, as well as a randomly selected recovery trial after each error. Half of all trials included at least one target and required a go response; the other half of the trials did not include either target and required that the subject withhold a response. In each block of 42 test trials, 7 trials included only the first target feature (e.g., X), 7 included only the second target feature (e.g., green), 7 included both targets, and the remaining 21 included no targets. Most important, this design includes neither an interstimulus contingency benefit nor a nontarget-response contingency benefit. In particular, the conditional probability of a target being present in a given dimension was balanced across the single- and redundant-target conditions (in each case, it was 0.5 ); thus, there was zero interstimulus contingency benefit. In addition, the probability that a go response should be made given the presence of a nontarget feature was equal to the baseline probability that a go response was correct (both were 0.5 ); thus, there was zero nontarget-response contingency benefit (see Mordkoff \& Yantis, 1991, for additional details).
Procedure. Each subject participated in two sessions conducted at similar times on consecutive days. During Session 1 , the subjects first completed a short practice block during which they were given bonus-point feedback after each trial. In total, Session 1 included one block of 20 trials and eight blocks of approximately 50 trials, of which only the last 4 were retained for analysis. Session 2 also began with a short block of 20 practice trials, followed by 14 blocks of testing trials. The first two full blocks of Session 2 were also considered practice. Data from all warm-up and recovery trials (i.e., the first five trials in a block and the trials immediately following each error) were also excluded from the analysis.

Each trial began with the presentation of a fixation cross at the center of the screen for $750 \mathrm{msec}$. After a 100 -msec blank interval, the test stimulus appeared. As soon as a response was made, whether correct or a false alarm, the stimulus was removed. If no response had been made by $1,000 \mathrm{msec}$, the test display was removed and the trial was considered a no-go. The intertrial interval was $2,200 \mathrm{msec}$.

A $700-\mathrm{Hz}$ tone sounded for $\mathbf{2 0 0} \mathrm{msec}$ following all false-alarm and miss errors. Three tones were played if the subject made an anticipation error by responding before the test stimulus appeared. (Anticipations were extremely rare-less than $0.01 \%$ overall-and will not be discussed further.) End-of-block feedback was displayed during an enforced 7-sec break; it consisted of mean RT in milliseconds, accuracy in percent, and total number of bonus points earned (or lost). The subjects received approximately 35 points for each correct go response, slightly more for a rapid response, and slightly less for a slow response. Fifteen points were awarded for a correct no-go, but 350 points were subtracted for any miss, false alarm, or anticipation response.

Data analysis. Four separate analyses were conducted. The first concerned simple redundancy gains in mean RT. This analysis revealed whether responses to redundant-target displays were significantly faster than those to single-target displays by comparing (within subjects) the mean RT on redundant-target trials with the mean RT on single-target trials.

The second analysis also examined redundancy gains, but compared the mean RT on redundant-target trials with the mean RT for the faster of the two types of single-target trial for each given subject. This fixed favored dimension test (see Biederman \& Checkosky, 1970; Miller \& Lopes, 1988; Mullin, Egeth, \& Mordkoff, 1988 ) is much more conservative than the simple redundancy-gain test: It corrects for artifactual redundancy gains arising because one dimension is processed more slowly than the other, which would affect the single-target mean to a greater extent than the redundanttarget mean.

The last two analyses checked for violations of the race-model inequality (for details, see Miller, 1982; Mordkoff \& Yantis, 1991). The first of these tests used raw (uncorrected) data; the second used data that had been corrected for fast guesses using the "twin-killing" method (see Eriksen, 1988; Miller \& Lopes, 1991). ${ }^{2}$ In both tests, the cumulative distribution functions (CDFs) were evaluated at the 10 th-95th percentiles at $5 \%$ intervals. Group plots were created by Vincentizing the data across subjects. Vincentizing is done by finding the value of $t$ corresponding to the $10 \mathrm{th}, 15 \mathrm{th}, 20 \mathrm{th}$ percentiles, and so on, separately for each subject, and then finding the mean value of $t$ across all subjects at each of the 18 quantiles. This procedure preserves the shape of all constituent distributions (Thomas \& Ross, 1980).

\section{Results}

The mean RT and accuracy data are shown in Table 1. Group plots of the (uncorrected) Vincentized data from Experiment 1 appear in Figure 1.

Redundancy gains. Mean RT on redundant-target trials was significantly less than mean RT on single-target trials. 
Table 1

Mean Response Time (RT, in Milliseconds) and Error Rates (Misses and False Alarms, in Percent) From Experiments 1-5

\begin{tabular}{|c|c|c|c|c|c|}
\hline & $\begin{array}{l}\text { Experiment } 1 \\
\text { Colored Letter }\end{array}$ & $\begin{array}{l}\text { Experiment } 2 \\
\text { Colored Frame } \\
\text { Around Letter }\end{array}$ & $\begin{array}{c}\text { Experiment } 3 \\
\text { Color Patch } \\
\text { and Letter }\end{array}$ & $\begin{array}{c}\text { Experiment } 4 \\
\text { Two Different } \\
\text { Letters }\end{array}$ & $\begin{array}{l}\text { Experiment } 5 \\
\text { Two Different } \\
\text { Color Patches }\end{array}$ \\
\hline \multicolumn{6}{|c|}{ Mean RT } \\
\hline Redundant Targets & 302 & 307 & 279 & 314 & 340 \\
\hline Single Target & 344 & 345 & 318 & 346 & 377 \\
\hline Redundancy Gain & $42 *$ & $38^{*}$ & $39 *$ & $32 *$ & $38 *$ \\
\hline Faster Single Target & 333 & 334 & 304 & 335 & 360 \\
\hline Conservative Redundancy Gain & $32 *$ & $27^{*}$ & $24^{*}$ & $20^{*}$ & $20^{*}$ \\
\hline \multicolumn{6}{|c|}{ Miss Errors } \\
\hline Single Target & 0.2 & 0.0 & 0.0 & 0.1 & 0.0 \\
\hline Redundant Targets & 0.1 & 0.1 & 0.0 & 0.1 & 0.1 \\
\hline \multicolumn{6}{|c|}{ False-Alarm Errors } \\
\hline & 1.3 & 1.3 & 2.0 & 2.0 & 3.5 \\
\hline
\end{tabular}

${ }^{*} p<.001$.

The mean redundancy gain was $41.9 \mathrm{msec}[t(11)=14.3$, $p<.001]$. The conservative test also showed a significant effect of redundant targets (see Table 1).

Tests of the race-model inequality. There was a wide range of values for which the race-model inequality was violated in Experiment 1 . This is evidenced by the placement of the curve representing the sum of the single-target CDFs below and to the right of the redundant-target CDF (see Figure 1). For 8 of the 18 quantiles tested in Experiment 1 , the observed violation was significant $(p<.05$, or better). The results using the data corrected for fast guessing produced similar results.
Mean accuracy. In general, the subjects were very accurate, with mean miss rates below $0.2 \%$ and false-alarm rates of $1.3 \%$ (Table 1). More important, the subjects did not make more miss errors on redundant-target trials than they did on single-target trials; thus, there is no evidence of a speed-accuracy tradeoff.

\section{Discussion}

The observation of significant violations of the racemodel inequality rules out all models that posit independent decisions concerning target-color presence and targetshape presence. Responses to displays that contain target

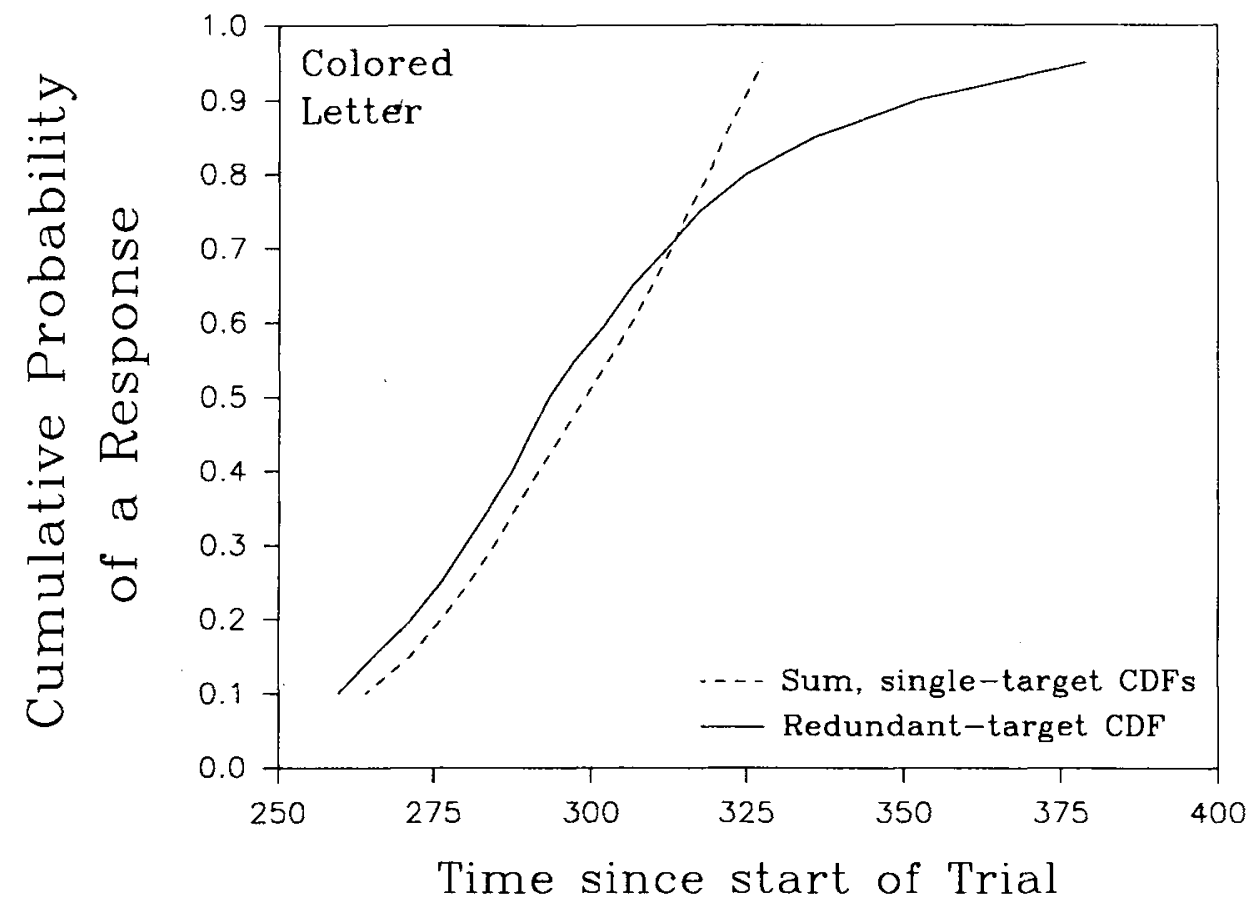

Figure 1. Group cumulative distribution functions for Experiment 1. (The race-model inequality is significantly violated at 8 of the 18 quantiles.) 
features in both perceptual dimensions are not activated by the faster of two independent processing channels; instead, the signals appear to combine their activations, causing responses that are faster than the fastest times ever observed with either target alone. Furthermore, because these violations were observed using an experimental design that lacks biased contingencies, an account of this task based on the interactive race model is also disconfirmed. Some form of coactivation appears to occur when attention must be divided between two distinct features of a single object.

\section{EXPERIMENTS 2 AND 3}

There are in fact two differences between the stimuli used in Experiment 1 and those used in previous redundanttarget experiments with spatially disparate objects. So far, we have focused on the fact that the target features used in Experiment 1 appeared as parts of a single perceptual object. The stimuli in Experiment 1 also differed from those in previous experiments in that the relevant features were in different dimensions (color and shape), whereas in all previous experiments there was only one relevant dimension (shape). Therefore, we cannot be sure that the critical aspect of Experiment 1 was that the target features were parts of the same perceptual object.

Experiments 2 and 3 disentangled these two possible accounts by repeating Experiment 1 with color and shape as the relevant features, but separating them in space. In Experiment 2, the shape was a white letter appearing at fixation, and the color was rendered as a nonwhite frame that surrounded the letter. As before, the target features were $X$ and green. Single-target trials in Experiment 2 included a white $\mathrm{X}$ surrounded by a purple frame, or a green frame surrounding a white $O$, and the redundanttarget display consisted of a white $\mathrm{X}$ surrounded by a green frame. In Experiment 3, we separated the shape and the color still further: The letter was white and appeared above fixation, while a colored square patch appeared below fixation.

\section{Method}

Experiments 2 and 3 were identical to Experiment 1 with the following exceptions. Twelve new subjects from the Johns Hopkins and UCSD subject pools participated in each experiment. The frame surrounding the central white letter in Experiment 2 subtended $2.10^{\circ} \times 1.34^{\circ}$ visual angle. In Experiment 3, the letter was presented $1.53^{\circ}$ above fixation and the color patch $\left(0.89^{\circ}\right.$ square $)$ was presented $1.53^{\circ}$ below fixation.

\section{Results}

The mean RT and accuracy data from Experiments 2 and 3 are shown in Table 1. Group plots of the (uncorrected) Vincentized data from Experiments 2 and 3 appear in Figures 2 and 3, respectively.

Redundancy gains. In both Experiments 2 and 3, mean RT on redundant-target trials was significantly less than mean RT on single-target trials. For Experiment 2, the redundancy gain was $37.8 \mathrm{msec}[t(11)=12.3, p<$
$.001]$; for Experiment 3, it was $38.5 \mathrm{msec}[t(11)=16.6$, $p<.0011$. The conservative redundancy-gain tests also showed a significant effect of redundant targets (see Table 1).

Tests of the race-model inequality. As in Experiment 1 , violations of the race-model inequality were observed over a wide range of values: The curve representing the sum of the single-target CDFs is below and to the right of the redundant-target CDF for both Experiment 2 (Figure 2) and Experiment 3 (Figure 3). For at least 6 of the 18 quantiles tested in each experiment, the observed violations were significant $(p<.05)$. Analyses using the corrected data yielded similar outcomes.

Mean accuracy. Again, the subjects were very accurate, with miss errors virtually absent and false-alarm rates of $1.3 \%$ and $2 \%$ for Experiments 2 and 3, respectively (Table 1).

\section{Discussion}

We noted that there were two possible accounts for the observed violations of the race-model inequality in Experiment 1: The violations might be attributed to how different perceptual dimensions are processed in vision, or they might be attributed to how decisions are made when both target features are parts of the same object. Experiments 2 and 3 rule out the latter account. At least in Experiment 3 the two target features were clearly parts of two different objects, yet significant violations of racemodel inequality were again observed.

Together, the results of Experiments 1-3 suggest that when targets must be detected from more than one perceptual dimension, activations from different dimensions may summate in generating a response. These results contrast sharply with those from previous experiments requiring the detection of a single stimulus feature appearing in either of two distinct objects. When subjects are required to detect a shape in either or both of two locations, for example, the data do not violate the race-model inequality when the experimental design includes no biased contingencies (Mordkoff \& Yantis, 1991). Thus, while the interactive race model may provide a comprehensive account of the results from tasks requiring spatially divided attention, the model cannot account for the results from tasks that require attention to be divided between separable stimulus dimensions. Furthermore, mere spatial separation is not sufficient to allow for separate activations; targets from different perceptual dimensions may also coactivate even when presented as parts of distinct objects. The number of dimensions from which targets must be detected appears to be much more important in determining divided-attention performance than the number of objects (or spatial areas) over which attention must be divided.

\section{EXPERIMENTS 4 AND 5}

The task used in Experiments 1-3 included a factor that could provide an alternative account of the discrepancy 


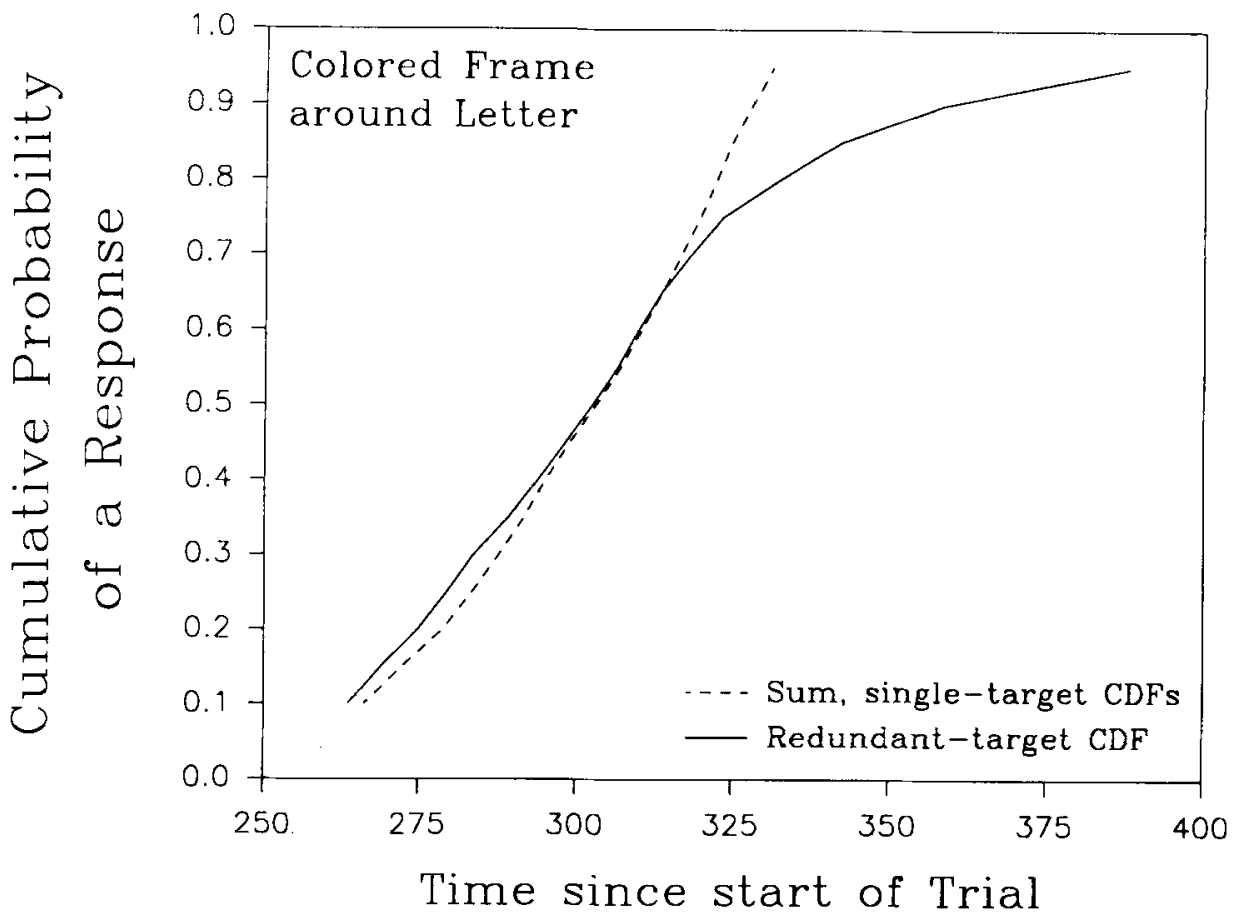

Figure 2. Group cumulative distribution functions for Experiment 2. (The race-model inequality is significantly violated at 9 of the 18 quantiles.)

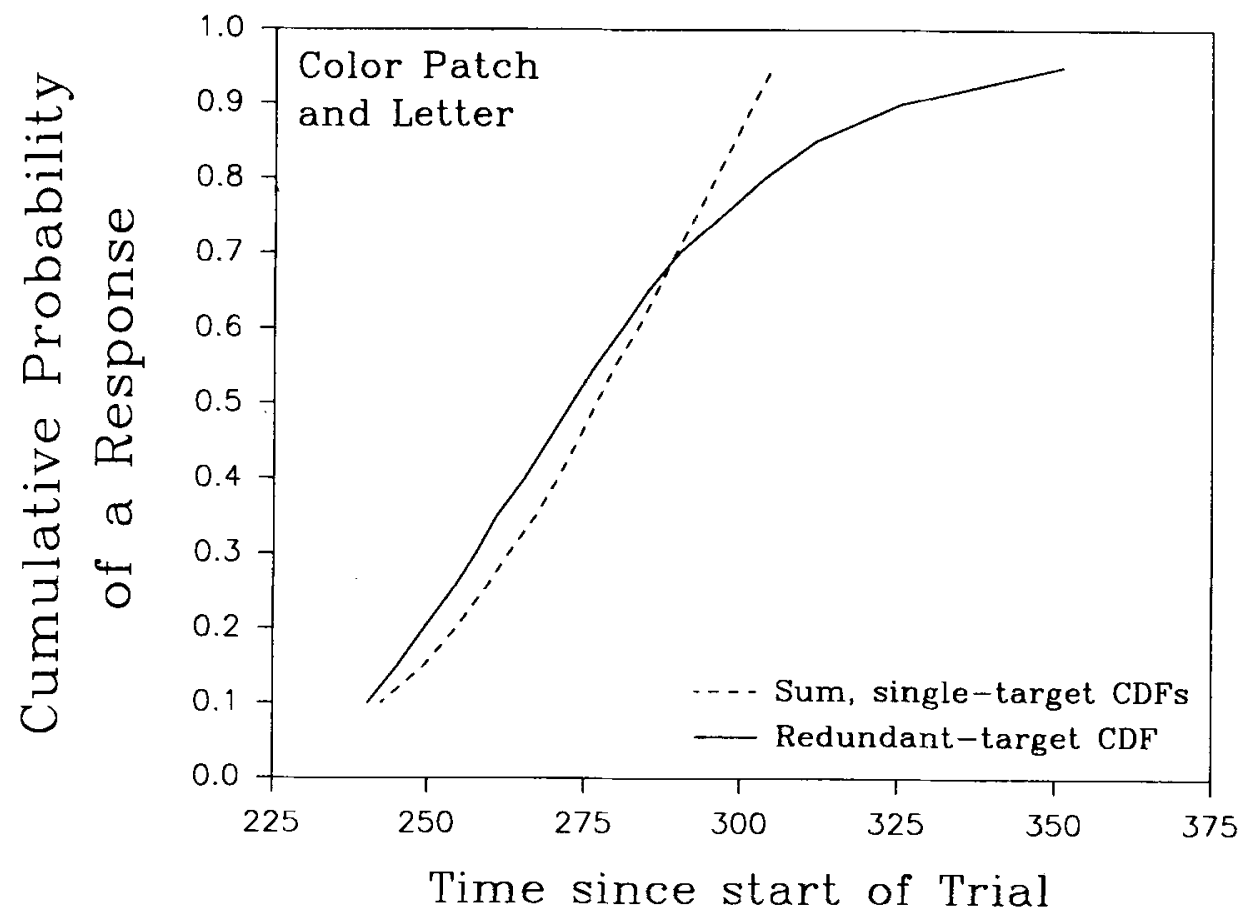

Figure 3. Group cumulative distribution functions for Experiment 3. (The race-model inequality is significantly violated at 6 of the 18 quantiles.) 
between the present results and earlier results (e.g., Mordkoff \& Yantis, 1991). This concerns the number of targets for which subjects had to search. In our earlier experiments, subjects were required to search for only one target feature (the shape X), whereas in the present experiments, the subjects had to search for both a color (green) and a shape (X). Thus, the difference in resultsnamely, satisfaction of the race-model inequality when attention is divided across spatial locations with only one dimension being task relevant, and violations of the racemodel inequality when attention must be divided between separable dimensions-may not be due to the singledimension versus multiple-dimension distinction. It may instead be due to the difference in task complexity as defined in terms of target-set size.

To test this alternative account, we conducted two additional experiments in which attention had to be divided between two different objects and the targets to be detected were within a single dimension. However, in contrast to our previous experiments examining withindimension divided attention (Mordkoff \& Yantis, 1991), these new experiments involved two different targets and four different distractors. Experiment 4 concerned only the shape dimension, and all stimuli were white letters. Experiment 5 concerned only the color dimension, and all stimuli were square patches of color. In both experiments, one target and two nontargets appeared only in the upper display location, and the other target and the other two nontargets appeared only in the lower location. This matches the conditions employed in the present Experiment 3, where one target letter and two nontarget letters appeared in the upper display location, and one target color and two nontarget colors appeared in the lower location.

If task complexity (i.e., number of targets) is the critical factor that accounts for the difference in results between our single- and multiple-dimension experiments, then Experiments 4 and 5 should exhibit violations of the race-model inequality similar to those observed in Experiments 1-3. In contrast, if the violations of the race-model inequality observed in Experiments 1-3 were due to the presentation of targets in two separable dimensions, then Experiments 4 and 5 should yield data that satisfy the racemodel inequality, because the experimental design included no biased contingencies and each involved only one perceptual dimension.

\footnotetext{
Method

Two new groups of 12 undergraduates participated in Experiments 4 and 5 . None had served in any of the previous experiments.

In Experiment 4, two different white letters appeared in each display. The letters were A, M, O, T, U, and X. Target and distractor sets were selected randomly and uniquely for each subject. A random set of three of these letters were selected to appear only in the upper display location, and one of them was designated as the first target; the other three letters only appeared in the lower location, and one of them was designated as the second target. Once selected, these sets remained fixed for the entire experiment. Each letter was $1.40^{\circ}$ tall and $0.89^{\circ}$ wide and was placed $1.53^{\circ}$ above or below fixation.
}

In Experiment 5, two different colors appeared in each display. The colors were blue, green, cyan, red, purple, and yellow (palette codes 9-14). Three colors appeared only in the upper location; the other three appeared only in the lower location. Each color patch was $0.89^{\circ} \times 0.89^{\circ}$ and was placed $1.53^{\circ}$ above or below fixation.

\section{Results}

A summary of the mean RT and accuracy data may be found in Table 1 . Both the standard and the conservative tests for a redundancy gain revealed significant differences in mean RT. In Experiment 4, the redundancy gain was $31.7 \mathrm{msec}[t(11)=7.8, p<.001]$. In Experiment 5 , the redundancy gain was $37.7 \mathrm{msec}[t(11)=9.6, p<.001]$. Figures 4 and 5 show the (uncorrected) Vincentized CDFs from Experiments 4 and 5, respectively. No significant violations of the race-model inequality were observed in either experiment; the few violations observed in Experiment 4 were all unreliable (all $t \mathrm{~s}<0.50$ ). An analysis of the error-corrected data yielded the same results.

\section{Discussion}

The results from Experiments 4 and 5 rule out the alternative, task-complexity account for Experiments 1-3 and provide additional support for the notion that dividing attention between separable dimensions is qualitatively different from dividing attention (only) between spatial locations. In particular, the results from Experiments 1-3 violated the race-model inequality at numerous quantiles, and these violations were often quite large. In contrast, the results from Experiment 4 showed only a few, small violations-none of which were significant-and those from Experiment 5 did not violate the race-model inequality at any quantile. Indeed, the results from Experiments 4 and 5 are quite similar to those from Experiment 1 of Mordkoff and Yantis (1991), which used the same experimental design but only one type of target.

\section{GENERAL DISCUSSION}

The data from experiments that require subjects to divide their attention between spatially disparate objects, with only one perceptual dimension being task relevant, do not violate the race-model inequality -as long as no biased contingencies are included in the experimental design (Mordkoff \& Yantis, 1991). This result is consistent with an interactive race model of divided attention. It has been observed in situations involving only one target feature (Mordkoff \& Yantis, 1991, Experiment 1; van der Heijden et al., 1984) and in situations involving more than one target (the present Experiments 4 and 5). Our previous work has concerned only letter shape; the present study has extended this finding to color.

In contrast, the results from experiments requiring attention to be divided between two separable dimensions, such as color and shape, significantly violate the racemodel inequality. This result has been observed in the absence of biased contingencies, ruling out an account of that task based on the interactive race model or any other separate-activations model. This conclusion may also ap- 


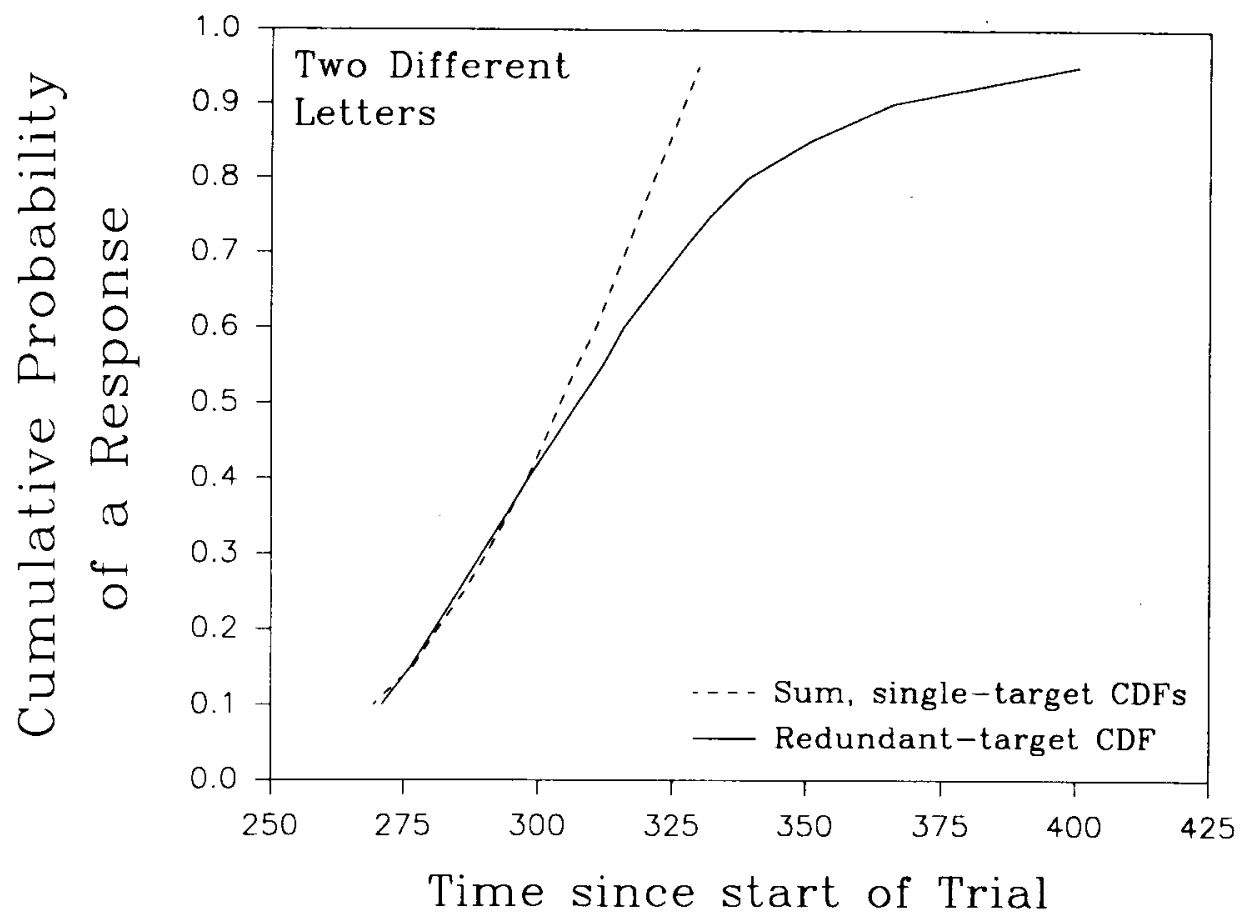

Figure 4. Group cumulative distribution functions for Experiment 4. (There are no significant violations of the race-model inequality.)

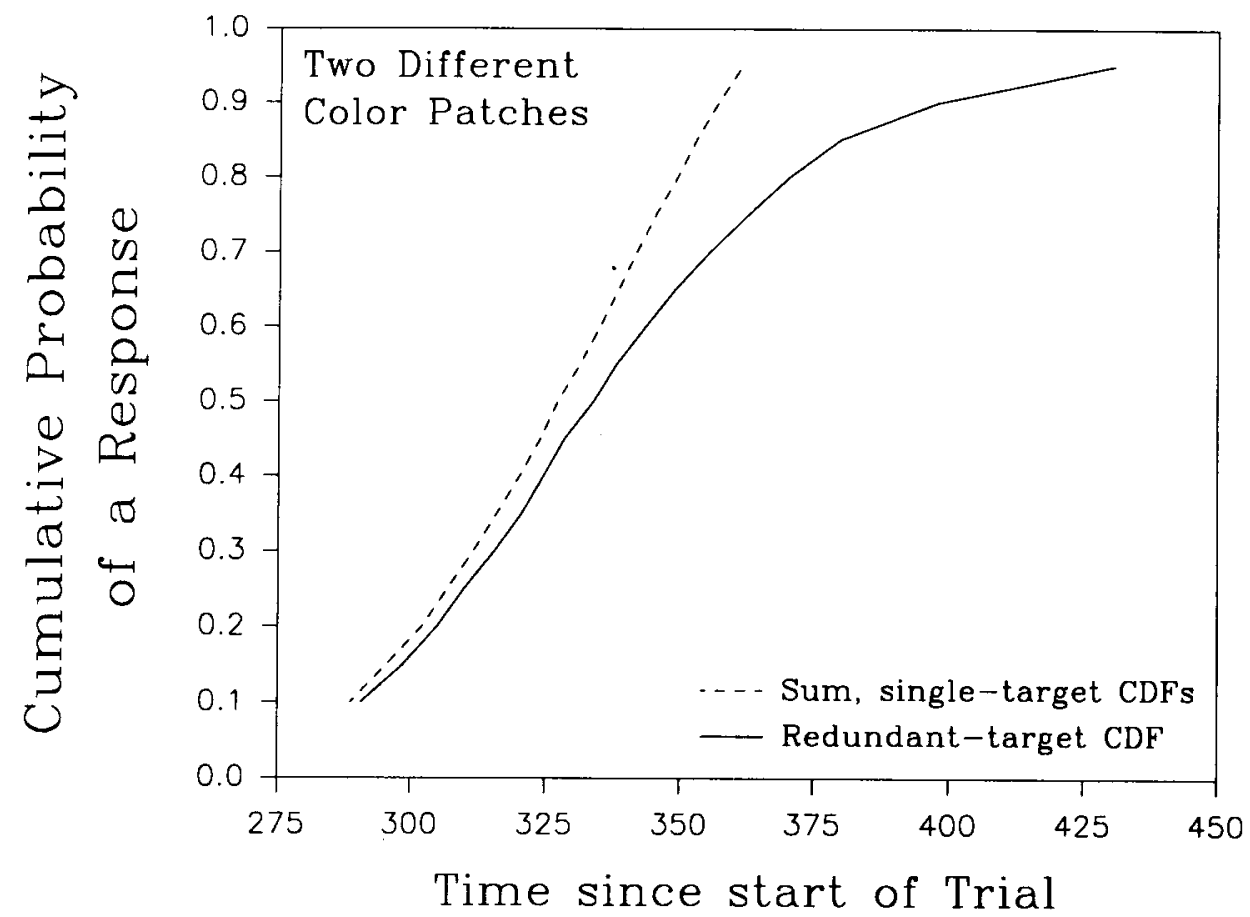

Figure 5. Group cumulative distribution functions for Experiment 5. (There are no violations of the race-model inequality.) 
ply to situations in which attention must be divided between sensory modalities (Miller, 1991) or when the task requires a multidimensional same/different judgment (Miller, 1978; but see Garner, 1988, and St. James \& Eriksen, 1990, for discussions of additional factors that arise in same/different tasks).

Taken together, these data suggest that the "race" posited by separate-activations models occurs only within perceptual modules (e.g., the set of processes that encodes visual shapes), and that coactivation occurs between modules when more than one dimension is task-relevant and both modules are processing a target. More precisely, each perceptual module appears to produce a single output code that enjoys the benefits of statistical facilitation on within-dimension, redundant-target trials; however, the outputs from separate dimensions coactivate to produce a target-present response on between-dimension, redundanttarget trials. These ideas are represented schematically in Figure 6. Note the $O R$ within each perceptual module that denotes the "finishing line" for a within-dimension race between redundant targets. This contrasts with the $\Sigma$ (between the modules) that marks where module outputs are summed (i.e., coactivate) on between-dimension, redundant-target trials.

The modular account suggested here is related to an argument made by Wolfe et al. (1990) on the basis of visualsearch experiments. These authors found that withindimension conjunction search (e.g., search for an object that is both green and red) is much more difficult than between-dimension conjunction search (e.g., search for an object that is both green and circular). From these data, Wolfe and colleagues argued that the serial stage of their guided search model may receive only one input from each perceptual dimension. Although the relation between the mechanisms underlying visual search and divided attention is not clear, the two notions of how perceptual modularity influences performance are not inconsistent.

\section{Implications for an Alternative Account}

Although violations of the race-model inequality rule out separate-activations or race models, they do not provide evidence concerning the specific type of processing dependence. For example, while the results from Experiment 1 are inconsistent with any model positing that the color and shape of a stimulus are processed in parallel and independently, the results do not necessarily imply that the color and shape are processed in parallel and coactivate a response. An alternative possibility is that subjects first search for the conjunction of target features (i.e., a green $\mathrm{X}$ ) and, failing to find it, check the two dimensions one at a time (see, e.g., Farell, 1984). Such a model would predict the violations of the race-model inequality observed in Experiment 1, because the target-features conjunction would always be found on redundant-target trials and never found on single-target trials.

However, this "conjunction-search" model (cf. Treisman \& Gelade, 1980; Mordkoff, Yantis, \& Egeth, 1990) may be ruled out because it cannot explain the similarity between Experiments 1 and 3. Recall that these experiments differed in terms of whether the two target features on redundant-target trials were parts of the same or two different objects: A redundant-target trial in Experiment 1 involved the presentation of a green $X$, whereas the same condition in Experiment 3 involved a white $X$ and a

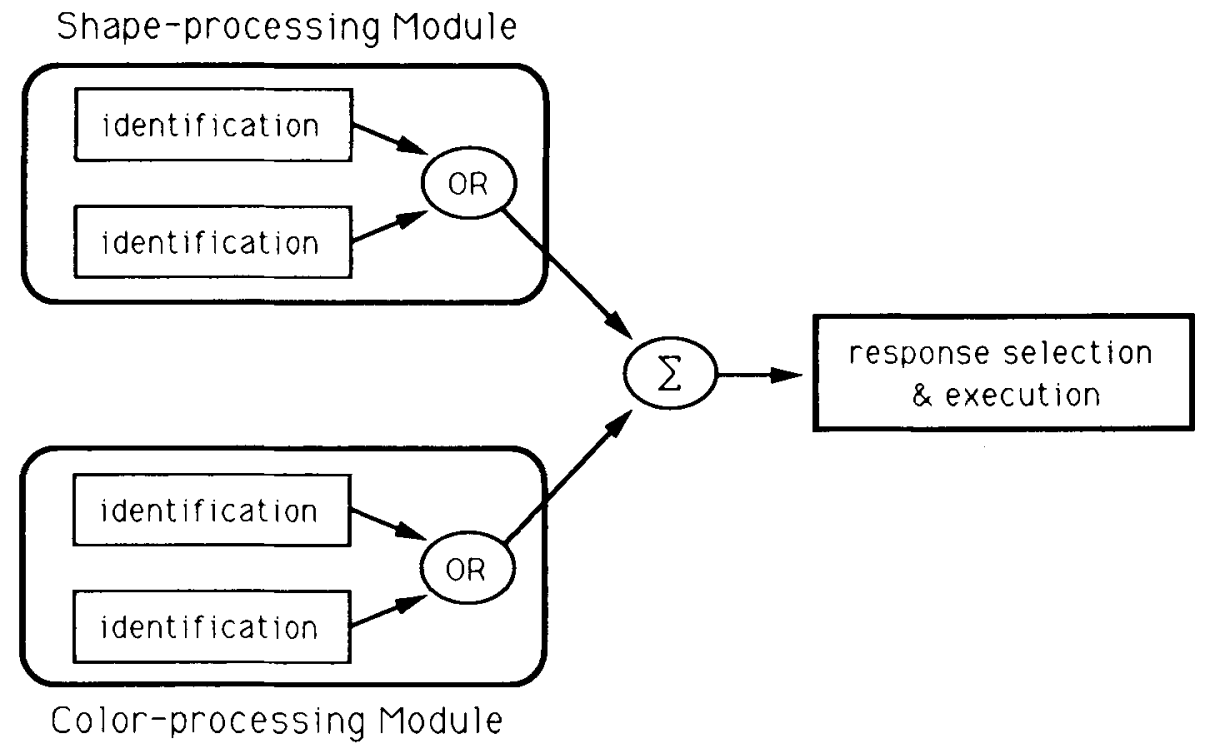

Figure 6. Schematic of a modular hybrid model of divided attention. (Under this model, the information from separable perceptual dimensions, such as color and shape, are processed by separate modules. Within each module, the codes from redundant targets race to activate a go output; the finishing line for each race is denoted by $O R$. Between the two modules, the codes from redundant targets coactivate; the point of summation is denoted by $\Sigma$.) 
spatially separate, green color patch. While the serial conjunction-search strategy could account for the results of Experiment 1, it would not be viable in Experiment 3. Yet, these two experiments produced remarkably similar results-the only difference being an overall, 25 - $\mathrm{msec}$ advantage for the responses in Experiment 3. This alternative account of our results must therefore be rejected.

\section{Conclusion}

In this paper, we have distinguished between two ways in which visual attention may be divided between sources of task-relevant information: Decisions may be required about the presence of features from a single dimension (appearing in any of several objects) or about the presence of features from separable dimensions (in one or more objects). Results from experiments examining the former, spatial form of divided attention support an interactive race model in which each target signal is processed separately and acts as an independent opportunity for a response to be activated (Mordkoff \& Yantis, 1991). Results from the experiments reported here, requiring that attention be divided between separable dimensions, suggest that coactivation occurs, with more than one target simultaneously activating a target-present response on redundant-target trials.

\section{REFERENCES}

Biederman, I., \& Checkosky, S. F. (1970). Processing redundant information. Journal of Experimental Psychology, 83, 486-490.

DUNCAN, J. (1984). Selective attention and the organization of visual information. Journal of Experimental Psychology: General, 113, 501-517.

EgETH, H. E. (1966). Parallel versus serial processes in multidimensional discrimination. Perception \& Psychophysics, 1, 245-252.

ERIKSEN, C. W. (1988). A source of error in attempts to distinguish coactivation from separate activation in the perception of redundant targets. Perception \& Psychophysics, 44, 191-193.

FARELL, B. (1984). Attention in the processing of complex visual displays: Detecting features and their combinations. Joumal of $E x-$ perimental Psychology: Human Perception \& Performance, 10, 40-64.

GARNER, W, R. (1962). Uncertainty and structure as psychological concepts. New York: Wiley.

GARNER, W. R. (1988). Facilitation and interference with a separable redundant dimension in stimulus comparison. Perception \& Psychophysics, 44, 321-330.

Grice, G. R., Canham, L., \& Boroughs, J. M. (1984). Combination rule for redundant information in reaction time tasks with divided attention. Perception \& Psychophysics, 35, 451-463.

Kahneman, D., Treisman, A., \& Gibs, B. (1992). The reviewing of object files: Object-specific integration of information. Cognitive Psychology, 24, 175-219.

Kanwisher, N., \& Driver, J. (1992). Objects, attributes, and visual attention: Which, what, and where. Current Directions in Psychological Science, 1, 26-31.

Kubovy, M., \& Cohen, D. (1991, November). Are texture segregation by form and color independent of each other? Paper presented at the 32nd Annual Meeting of the Psychonomic Society, San Francisco, CA.

Miller, J. (1978). Multidimensional same-different judgments: Evidence against independent comparisons of dimensions. Journal of Experimental Psychology: Human Perception \& Performance, 4, 411 422.
Miller, J. (1982). Divided attention: Evidence for coactivation with redundant signals. Cognitive Psychology, 14, 247-279.

MilLER, J. (1991). Channel interaction and the redundant-targets effect in bimodal divided attention. Journal of Experimental Psychology: Human Perception \& Performance, 17, 160-169.

MILLER, J., \& LOPES, A. (1988). Testing race models by estimating the smaller of two true means or median reaction times. Perception \& Psychophysics, 44, 513-524.

Miller, J., \& LOPES, A. (1991). Bias produced by fast guessing in distribution-based tests of race models. Perception \& Psychophysics, 50, 584-590.

MoRdKOFF, J. T., \& YANTIS, S. (1991). An interactive race model of divided attention. Journal of Experimental Psychology: Human Perception \& Performance, 17, 520-538.

Mordkoff, J. T., YANTIS, S., \& EgETH, H. E. (1990). Detecting conjunctions of color and form in parallel. Perception \& Psychophysics, 48, 157-168.

Mulun, P., Egeth, H. E., \& Mordkoff, J. T. (1988). Redundanttarget detection and processing capacity: The problem of positional preferences. Perception \& Psychophysics, 43, 607-610.

RAAB, D. (1962). Statistical facilitation of simple reaction time. Transactions of the New York Academy of Sciences, 43, 574-590.

ST. JAMES, J. D., \& ERIKSEN, C. W. (1990). Response competition produces a "fast same effect" in same-different judgments. In G. R. Lockhead \& J. R. Pomerantz (Eds.), The perception of structure (pp. 157168). Washington, DC: American Psychological Association.

Tномаs, E. A. C., Ross, B. H. (1980). On appropriate procedures for combining probability distributions within the same family. Journal of Mathematical Psychology, 21, 136-152.

Treisman, A. M., \& Gelade, G. (1980). A feature integration theory of attention. Cognitive Psychology, 12, 97-136.

van der Heijden, A. H. C., La Hei, W., \& Boer, J. P. A. (1983). Parallel processing of redundant targets in simple search tasks. Psychological Research, 45, 235-254.

van der Heuden, A. H. C., Schreuder, R., Maris, L., \& NeErincx, M. (1984). Some evidence for correlated separate activations in a simple letter-detection task. Perception \& Psychophysics, 36, 577-585.

Wolfe, J. M., Yu, K. P., Stewart, M. I., Shorter, A. D., FriedmanHill, S. R., \& Cave, K. R. (1990). Limitations on the parallel guidance of visual search: Color $\times$ color and orientation $x$ orientation conjunctions. Joumal of Experimental Psychology: Human Perception \& Performance, 16, 879-892.

YANTIS, S. (1992). Multielement visual tracking: Attention and perceptual organization. Cognitive Psychology, 24, 295-340.

\section{NOTES}

1. Some previous studies have required that attention be divided between two or more dimensions, but these tasks have involved same/different judgments, responses concerning texture segregation, and/or were not analyzed for violations of the race-model inequality (e.g., Biederman \& Checkosky, 1970; Egeth, 1966; Kubovy \& Cohen, 1991; Miller, 1978). Furthermore, as shown by Mordkoff and Yantis (1991), many of the early studies included biased contingencies within their designs. Thus, these data will not be reviewed in detail.

2. Under the twin-killing method, the correct-response RT that is nearest in value to each false-alarm RT is removed from the corresponding go distribution. This correction operates on the assumption that all false alarms are actually fast-guess responses - that is, because the probability of guessing correctly is 0.5 , for each fast guess that turns out to be an error, there should be a fast guess that turned out to be correct.

(Manuscript received June 16, 1992; revision accepted for publication September 9, 1992.) 